Article

\title{
Type II Topp-Leone Inverted Kumaraswamy Distribution with Statistical Inference and Applications
}

\author{
Ramadan A. ZeinEldin ${ }^{1,2}$, Farrukh Jamal ${ }^{3}{ }^{\circledR}$, Christophe Chesneau ${ }^{4, *}$ and Mohammed Elgarhy ${ }^{5}$ \\ 1 Deanship of Scientific Research, King AbdulAziz University, Jeddah 21589, Saudi Arabia; \\ rzainaldeen@kau.edu.sa \\ 2 Faculty of Graduate Studies for Statistical Research, Cairo University, Al Orman, Giza Governorate 12613, \\ Egypt \\ 3 Department of Statistics, Govt. S.A Postgraduate College Dera Nawab Sahib, Bahawalpur, Punjab 63100, \\ Pakistan; drfarrukh1982@gmail.com \\ 4 Department of Mathematics, Université de Caen, LMNO, Campus II, Science 3, Caen 14032, France \\ 5 Valley High Institute for Management Finance and Information Systems, Obour, Qaliubia 11828, Egypt; \\ m_elgarhy85@sva.edu.eg \\ * Correspondence: christophe.chesneau@unicaen.fr; Tel.: +33-02-3156-7424
}

Received: 5 November 2019; Accepted: 26 November 2019; Published: 28 November 2019

\begin{abstract}
In this paper, we present and study a new four-parameter lifetime distribution obtained by the combination of the so-called type II Topp-Leone-G and transmuted-G families and the inverted Kumaraswamy distribution. By construction, the new distribution enjoys nice flexible properties and covers some well-known distributions which have already proven themselves in statistical applications, including some extensions of the Bur XII distribution. We first present the main functions related to the new distribution, with discussions on their shapes. In particular, we show that the related probability density function is left, right skewed, near symmetrical and reverse J shaped, with a notable difference regarding the right tailed, illustrating the flexibility of the distribution. Then, the related model is displayed, with the estimation of the parameters by the maximum likelihood method and the consideration of two practical data sets. We show that the proposed model is the best one in terms of standard model selection criteria, including Akaike information and Bayesian information criteria, and goodness of fit tests against three well-established competitors. Then, for the new model, the theoretical background on the maximum likelihood method is given, with numerical guaranties of the efficiency of the estimates obtained via a simulation study. Finally, the main mathematical properties of the new distribution are discussed, including asymptotic results, quantile function, Bowley skewness and Moors kurtosis, mixture representations for the probability density and cumulative density functions, ordinary moments, incomplete moments, probability weighted moments, stress-strength reliability and order statistics.
\end{abstract}

Keywords: type II Topp-Leone distribution; inverted Kumaraswamy distribution; estimation; simulation; data analysis.

MSC: 60E05; 62E15; 62F10

\section{Introduction}

The detailed explanation of a lifetime phenomenon often follows from a deep data analysis based on a well-chosen statistical model. Since the "universal best model" remains an Utopian idea, a lot of effort has been put into the construction of models with different features, involving 
the development of new probability distributions. The mathematical techniques for creating new probability distributions are numerous. A common technique is to introduce one or several additional tuning parameters to a standard probability distribution, with the aim to improve it, in the theoretical and practical sense. We refer the reader to the following families of distributions: the exponentiated-G (or exp-G) family [1], the beta-G family [2], the Marshall-Olkin-G family [3], the gamma-G family [4], the Kumaraswamy-G family [5], the type I half-logistic-G family [6], the transmuted-G family [7], the odd power Cauchy family [8], the exponentiated generalised Topp-Leone-G family [9], the type II Topp-Leone-G family [10] and the type II general inverse exponential-G family [11].

Let us now show some basics of the type II Topp-Leone-G family of distributions introduced by [10]. The corresponding cumulative distribution function (cdf) and probability density function (pdf) are, respectively, given by

$$
F(x ; \alpha, \xi)=1-\left\{1-[G(x ; \xi)]^{2}\right\}^{\alpha}, \quad x \in \mathbb{R}
$$

and

$$
f(x ; \alpha, \xi)=2 \alpha g(x ; \xi) G(x ; \xi)\left\{1-[G(x ; \xi)]^{2}\right\}^{\alpha-1}, \quad x \in \mathbb{R}
$$

where $\alpha>0, G(x ; \xi)$ denotes a baseline cdf of a continuous distribution which may depend on a parameter vector $\xi$ and $g(x ; \xi)$ denotes the corresponding pdf. In addition to the special cases presented in [10], recent studies have highlighted the qualities of this family, and its ability to fit data sets by considering new baseline $\operatorname{cdf} G(x ; \xi)$. See, for instance, $[12,13]$ with the consideration of the generalised Rayleigh and Rayleigh distributions as baselines, respectively. There was, however, an area that needed to be explored by considering other kinds of $G(x ; \xi)$, which constituted the first piece of the idea of this study.

On the other side of things, with the aim to analyze lifetime data sets at their best, a new two-parameter distribution was introduced by [14]. It is called the inverted Kumaraswamy distribution. The corresponding cdf and pdf are, respectively, given by

$$
G_{*}(x ; a, b)=\left[1-(1+x)^{-a}\right]^{b}, \quad x>0
$$

and

$$
g_{*}(x ; a, b)=a b(1+x)^{-a-1}\left[1-(1+x)^{-a}\right]^{b-1}, \quad x>0,
$$

where $a, b>0$. This distribution, literally constructed from the inverse of a random variable following the so-called Kumaraswamy distribution minus one (see [15]), has been proven to be very rich. In particular, deep connexions exist with the so-called Lomax, inverted beta, log-logistic, inverted Weibull and generalised exponential distributions. However, a slight lack of flexibility can be seen in [14] (Figure 2): the hazard rate function seems to be limited in terms of curvatures (no J shape, no upside-down bathtub shape...). An immediate, elegant way to solve this problem is to extend it by using the simple transmuted technique proposed by [7]. Hence, the corresponding cdf and pdf are, respectively, given by

$$
\begin{aligned}
G(x ; a, b, \lambda) & =G_{*}(x ; a, b)\left[1+\lambda-\lambda G_{*}(x ; a, b)\right] \\
& =\left[1-(1+x)^{-a}\right]^{b}\left\{1+\lambda-\lambda\left[1-(1+x)^{-a}\right]^{b}\right\}
\end{aligned}
$$

and

$$
\begin{aligned}
g(x ; a, b, \lambda) & =g_{*}(x ; a, b)\left[1+\lambda-2 \lambda G_{*}(x ; a, b)\right] \\
& =a b(1+x)^{-a-1}\left[1-(1+x)^{-a}\right]^{b-1}\left\{1+\lambda-2 \lambda\left[1-(1+x)^{-a}\right]^{b}\right\}
\end{aligned}
$$


with $\lambda \in[-1,1]$. To the best of our knowledge, this remains a new three-parameter lifetime distribution in the literature. This constitutes the last piece of idea of the study.

That is, we introduce the type II Topp-Leone (transmuted) inverted Kumaraswamy (TIITLIK) distribution defined by combining the type II Topp-Leone-G family and the transmuted inverted Kumaraswamy distribution. We aim to offer a new ultra flexible four-parameter lifetime distribution, combining the qualities of these parents distributions, with a high potential of applicability. New statistical models for sophisticated data sets are the perspectives.

The plan of the paper is the following. In Section 2, we introduce the TIITLIK distribution, its main functions and some graphics illustrating the behaviour of the corresponding probability density and hazard rate functions. In Section 3, by the means of two practical data sets and the consideration of the maximum likelihood method, we show that the TIITLIK distribution fits better than well-established and modern adversary models. The details on the maximum likelihood method in the context of the TIITLIK distribution are given in Section 4, including a simulation study to show the nice numerical performances of the estimates. Section 5 presents the mathematical properties of the TIITLIK distribution, including asymptotes and critical points of the corresponding pdf and hrf, the quantile function, mixture representations, ordinary and central moments, incomplete moments, weighted probability moments, stress-strength reliability parameter and order statistics. Finally, some concluding remarks are given in Section 6.

\section{TIITLIK Distribution}

By adopting the notations above, the cdf of the TIITLIK distribution is given by

$$
\begin{aligned}
F(x ; \alpha, a, b, \lambda) & =1-\left\{1-[G(x ; a, b, \lambda)]^{2}\right\}^{\alpha} \\
& =1-\left\{1-\left[1-(1+x)^{-a}\right]^{2 b}\left\{1+\lambda-\lambda\left[1-(1+x)^{-a}\right]^{b}\right\}^{2}\right\}^{\alpha}, \quad x>0,
\end{aligned}
$$

with $\alpha, a, b>0$ and $\lambda \in[-1,1]$. To illustrate the richness of this cdf, some special cases are described below. When $\alpha=1$, we get the cdf of the two exponentiated, transmuted, inverted Kumaraswamy distributions with parameters $a, b$ and $\lambda$. When $\lambda=0$, we get the cdf of the Kumaraswamy inverted Kumaraswamy distribution with parameters $\alpha, a$ and $2 b$. When $\alpha=1$ and $\lambda=0$, we get the cdf of the inverted Kumaraswamy distribution with parameters $a$ and $2 b$. All these special cases contain several notable special cases themselves.

The survival function (sf) of the TIITLIK distribution is given by

$$
\begin{aligned}
R(x ; \alpha, a, b, \lambda) & =1-F(x ; \alpha, a, b, \lambda) \\
& =\left\{1-\left[1-(1+x)^{-a}\right]^{2 b}\left\{1+\lambda-\lambda\left[1-(1+x)^{-a}\right]^{b}\right\}^{2}\right\}^{\alpha}, \quad x>0 .
\end{aligned}
$$

Upon differentiation of $F(x ; \alpha, a, b, \lambda)$, the corresponding pdf is given by

$$
\begin{aligned}
& f(x ; \alpha, a, b, \lambda)=2 \alpha g(x ; a, b, \lambda) G(x ; a, b, \lambda)\left\{1-[G(x ; a, b, \lambda)]^{2}\right\}^{\alpha-1} \\
& =2 \alpha a b(1+x)^{-a-1}\left[1-(1+x)^{-a}\right]^{2 b-1}\left\{1+\lambda-2 \lambda\left[1-(1+x)^{-a}\right]^{b}\right\} \times \\
& \left\{1+\lambda-\lambda\left[1-(1+x)^{-a}\right]^{b}\right\}\left\{1-\left[1-(1+x)^{-a}\right]^{2 b}\left\{1+\lambda-\lambda\left[1-(1+x)^{-a}\right]^{b}\right\}^{2}\right\}^{\alpha-1} \\
& \quad x>0 .
\end{aligned}
$$


The corresponding hazard rate function (hrf) and cumulative hazard rate function (chrf) are, respectively, given by

$$
\begin{aligned}
& h(x ; \alpha, a, b, \lambda)=\frac{f(x ; \alpha, a, b, \lambda)}{R(x ; \alpha, a, b, \lambda)} \\
& =2 \alpha a b(1+x)^{-a-1}\left[1-(1+x)^{-a}\right]^{2 b-1}\left\{1+\lambda-2 \lambda\left[1-(1+x)^{-a}\right]^{b}\right\} \times \\
& \left\{1+\lambda-\lambda\left[1-(1+x)^{-a}\right]^{b}\right\}\left\{1-\left[1-(1+x)^{-a}\right]^{2 b}\left\{1+\lambda-\lambda\left[1-(1+x)^{-a}\right]^{b}\right\}^{2}\right\}^{-1}, \\
& \quad x>0
\end{aligned}
$$

and

$$
\begin{aligned}
& H(x ; \alpha, a, b, \lambda)=-\log [R(x ; \alpha, a, b, \lambda)] \\
& =-\alpha \log \left\{1-\left[1-(1+x)^{-a}\right]^{2 b}\left\{1+\lambda-\lambda\left[1-(1+x)^{-a}\right]^{2 b}\right\}\right\}, \quad x>0 .
\end{aligned}
$$

Figure 1 presents plots of the pdf and hrf of the TIITLIK distribution for fixed values of $\alpha, a, b$ and $\lambda$, showing a great diversity in terms of curvature. In particular, the plots of the pdf is left, right skewed, near symmetrical and reverse J shaped, with a notable difference on the right tailed-ness. The plots of the hrf are increasing, decreasing and upside-down bathtub (contrary to the former inverted Kumaraswamy distribution; see [14] (Figure 2)). These facts ensure a great ability of the related TIITLIK model to fit a wide variety of practical data sets. This aspect will be developed in detail in Section 3.
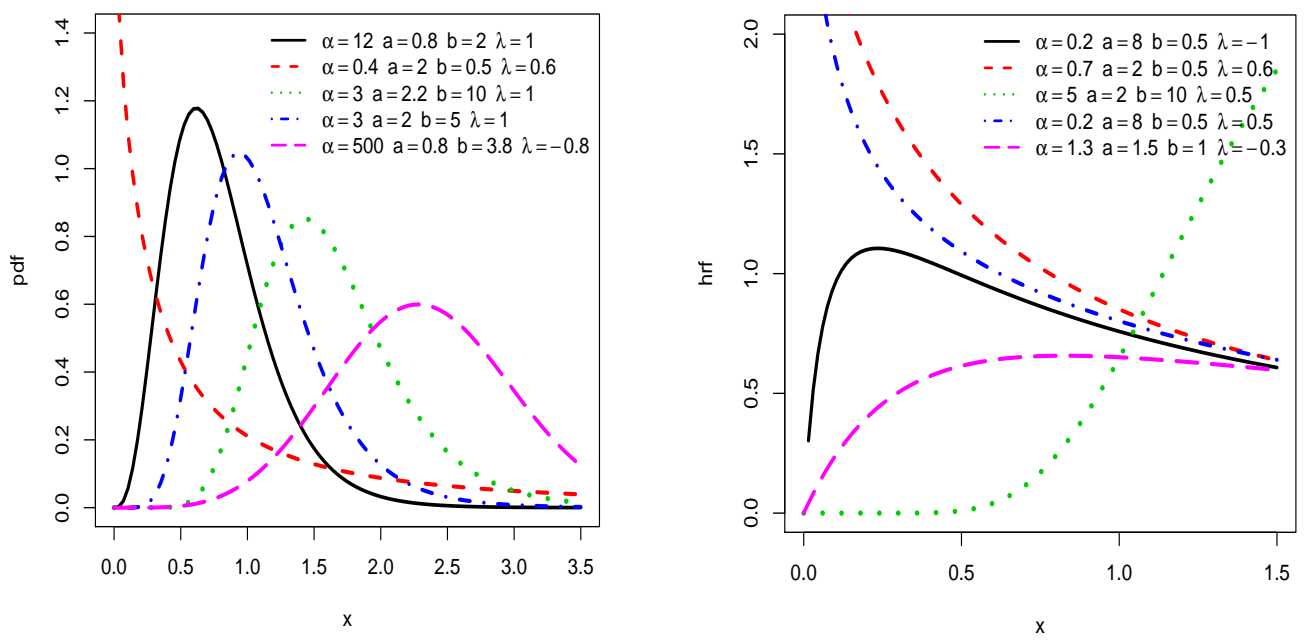

Figure 1. Plots of some probability density functions (pdfs) and hazard rate functions (hrfs) of the type II Topp-Leone (transmuted) inverted Kumaraswamy (TIITLIK) distribution.

\section{Applications}

We claim that the TIITLIK distribution has a high potential of applicability. In order to illustrate this claim, we analysed two practical data sets, with fair comparison to useful models in the literature, and discussions. Thus, we compared the TIHLIK distribution with the inverted Kumaraswamy (IK) distribution [14], generalised inverted Kumaraswamy (GIK) distribution by [16], Marshall-Olkin extended inverted Kumaraswamy (MOEIK) distribution [17] and Topp-Leone generalised inverted Kumaraswamy (TLGIK) distribution [18]. We refer to these papers for the exact definitions of the corresponding pdfs. 
We estimated the parameters of the corresponding models by the maximum likelihood method. The estimates, called maximum likelihood estimates (MLEs), were computed using R software with the library AdequacyModel, in which the function goodness. fit was used. We refer to Section 4 for the definitions and theoretical background of the MLEs in the context of the TIITLIK model.

The following statistical measures were calculated: log-likelihood $(\hat{\ell})$, Akaike information criterion (AIC), Bayesian information criterion (BIC), Anderson-Darling statistic ( $\mathrm{A}^{*}$ ) and Cramer-von Mises statistic $\left(\mathrm{W}^{*}\right)$. The lower the values of these criteria, the better the fit. We also provide the value for the Kolmogorov-Smirnov (KS) statistic and its $p$-value.

The first data set (data set 1): The first data set, given in [19], represents the annual maximum precipitation (in inches) for one rain gauge in Fort Collins (Colorado, USA) from 1900 through 1999. The heading of the data is as follows: $239,232,434,85,302,174 \ldots$

The second data set (data set 2): The second data set consists of annual maximum daily precipitation (in unit: $\mathrm{mm}$ ) at Busan(Korea) from 1904 through 2011 period. The data set has recently been used by [20]. The heading of the data is as follows: $24.8,140.9,54.1,153.5,47.9,165.5 \ldots$

Some descriptives statistics of these two data sets are given in Table 1. The TTT plots and boxplots for data sets 1 and 2 are shown in Figures 2 and 3, respectively. From the both TTT plots, we see a concave curve, indicating that the hrf behind the data is possibly increasing. This specificity also belongs to the hrf for the TIITLIK distribution for some values, justifying its consideration for these data sets. Further detail on the TTT plots can be found in [21]. MLEs and their standard errors (in parentheses) are presented in Tables 2 and 3 for data sets 1 and 2, respectively. The $-\hat{\ell}$, AIC, $\mathrm{BIC}, \mathrm{W}^{*}, \mathrm{~A}^{*}, \mathrm{KS}$ and $p$-value values are provided in Tables 4 and 5 for data sets 1 and 2, respectively. In all cases, we see that the TIITLIK is the best. To illustrate this numerical fact with graphics, Figures 4 and 5 present the empirical and estimated TIITLIK, hrfs and chrfs for data sets 1 and 2, respectively. Also, Figures 6 and 7 show the estimated pdfs for data sets 1 and 2, respectively. For these four last figures, nice fits are observed for the TIITLIK distribution, attesting its applicability for these data sets.

Table 1. A brief statistical description of data sets 1 and 2 .

\begin{tabular}{lcccccc}
\hline & $\mathbf{n}$ & Mean & Median & Standard Deviation & Skewness & Kurtosis \\
\hline Data set 1 & 100 & 175.67 & 158 & 83.17 & 1.32 & 1.71 \\
Data set 2 & 105 & 144.6 & 131.6 & 66.18 & 0.93 & 0.73 \\
\hline
\end{tabular}
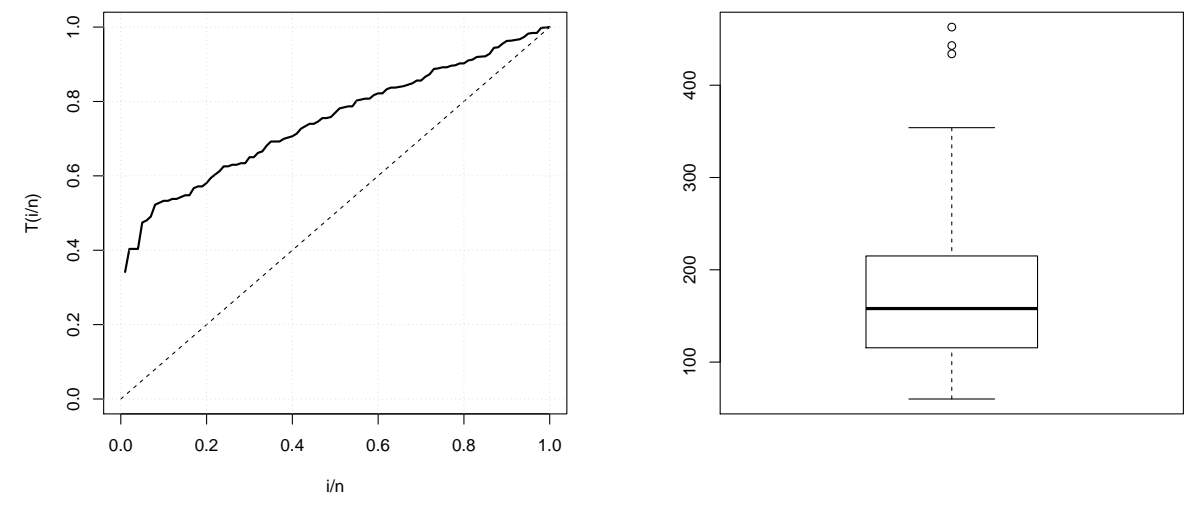

Figure 2. TTT plot and box plot for data set 1 . 

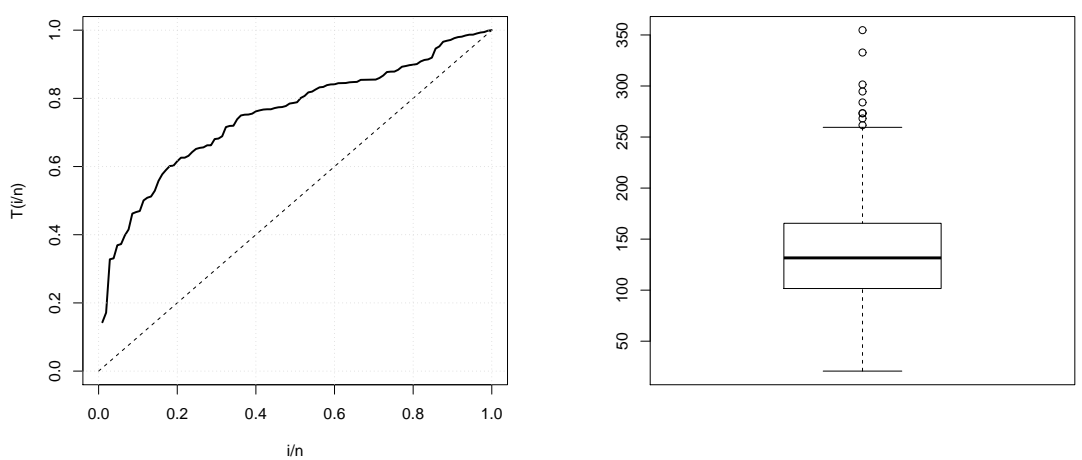

Figure 3. TTT plot and box plot for data set 2 .

Table 2. Maximum likelihoods (MLEs) with their standard errors for data set 1 .

\begin{tabular}{ccccccc}
\hline Model & $\boldsymbol{\alpha}$ & $\boldsymbol{a}$ & $\boldsymbol{b}$ & $\boldsymbol{\lambda}$ & $\boldsymbol{\beta}$ & $\boldsymbol{\theta}$ \\
\hline TIITLIK & 6.8935 & 0.7683 & 86.5351 & 0.9847 & - & - \\
& $(1.0629)$ & $(0.1939)$ & $(2.2302)$ & $(0.3637)$ & - & - \\
TLGIK & 1.0146 & - & - & 1.3053 & 89.8279 & 56.2739 \\
& $(3.6755)$ & - & - & $(4.6934)$ & $(4.1124)$ & $(3.8161)$ \\
MOEIK & 2.5225 & - & - & 566.2283 & 576.4857 & - \\
& $(0.1234)$ & - & - & $(25.8570)$ & $(27.8788)$ & - \\
GIK & 722.6636 & - & - & - & 0.5796 & 2.3328 \\
& $(9.2161)$ & - & - & - & $(1.3594)$ & $(5.4708)$ \\
IK & - & 0.7465 & 32.1198 & - & - & - \\
& - & $(0.0409)$ & $(6.0239)$ & - & - & - \\
\hline
\end{tabular}

Table 3. MLEs with their standard errors for data set 2 .

\begin{tabular}{ccccccc}
\hline Model & $\boldsymbol{\alpha}$ & $\boldsymbol{a}$ & $\boldsymbol{b}$ & $\boldsymbol{\lambda}$ & $\boldsymbol{\beta}$ & $\boldsymbol{\theta}$ \\
\hline TIITLIK & 35.0662 & 0.6705 & 30.7472 & -0.7928 & - & - \\
& $(3.0642)$ & $(0.1709)$ & $(1.1182)$ & $(0.1795)$ & - & - \\
TLGIK & 1.8475 & - & - & 0.5927 & 82.5348 & 6.8899 \\
& $(0.5184)$ & - & - & $(0.1581)$ & $(7.0094)$ & $(7.3323)$ \\
\multirow{2}{*}{ MOEIK } & 1.8704 & - & - & 99.9520 & 79.2607 & - \\
& $(0.0934)$ & - & - & $(5.5420)$ & $(6.7107)$ & - \\
\multirow{2}{*}{ GIK } & 351.3337 & - & - & - & 0.6904 & 1.8419 \\
& $(6.0827)$ & - & - & - & $(3.1515)$ & $(8.3987)$ \\
IK & - & 1.1921 & 241.3032 & - & - & - \\
& - & $(0.0554)$ & $(8.3953)$ & - & - & - \\
\hline
\end{tabular}

Table 4. Statistical measures for data set 1 .

\begin{tabular}{cccccccc}
\hline Model & $-\hat{\ell}$ & AIC & BIC & $\mathbf{W}^{*}$ & $\mathbf{A}^{*}$ & KS & $p$-Value (KS) \\
\hline TIITLIK & 565.9951 & 1139.9900 & 1150.4110 & 0.0377 & 0.2893 & 0.0458 & 0.9847 \\
TLGIK & 567.5158 & 1143.0320 & 1153.4520 & 0.0644 & 0.4290 & 0.0519 & 0.9502 \\
MOEIK & 580.6865 & 1167.3730 & 1175.1890 & 0.0424 & 0.3156 & 0.1544 & 0.0169 \\
GIK & 593.7943 & 1193.5890 & 1201.4040 & 0.0472 & 0.3960 & 0.2024 & 0.0005 \\
IK & 644.0206 & 1292.0410 & 1297.2520 & 0.0410 & 0.3652 & 0.3018 & 0.00000002 \\
\hline
\end{tabular}


Table 5. Statistical measures for data set 2 .

\begin{tabular}{cccccccc}
\hline Model & $-\hat{\ell}$ & AIC & BIC & $\mathbf{W}^{*}$ & $\mathbf{A}^{*}$ & KS & $p$-Value (KS) \\
\hline TIITLIK & 582.0684 & 1172.1370 & 1182.7530 & 0.1458 & 0.8300 & 0.0821 & 0.5000 \\
TLGIK & 600.0486 & 1208.0970 & 1218.7130 & 0.5001 & 2.8905 & 0.1202 & 0.0958 \\
MOEIK & 610.8292 & 1227.6580 & 1235.6200 & 0.1624 & 0.9319 & 0.2033 & 0.0003 \\
GIK & 616.0245 & 1238.0490 & 1246.0110 & 0.5327 & 3.0846 & 0.1999 & 0.0004 \\
IK & 620.0272 & 1244.0540 & 1249.3620 & 0.4794 & 2.7732 & 0.2147 & 0.0001 \\
\hline
\end{tabular}
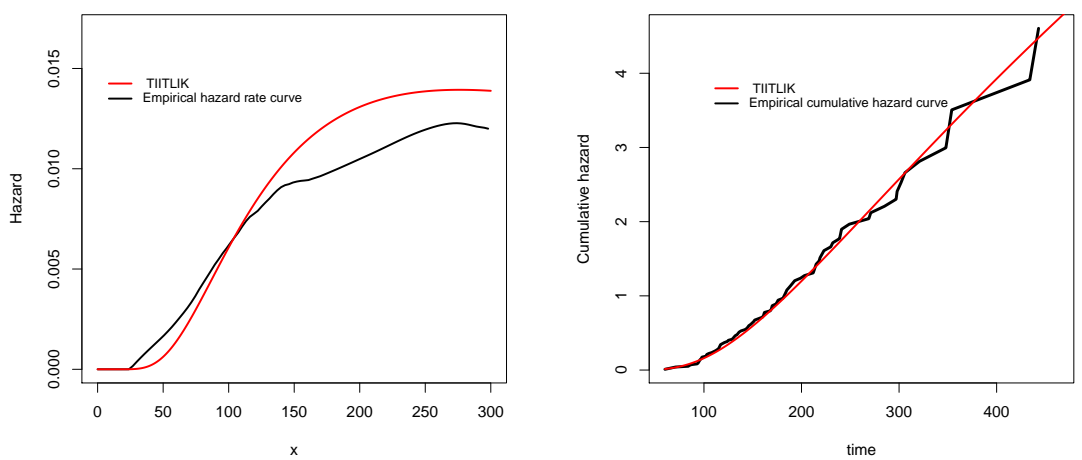

Figure 4. Plots of the empirical and estimated TIITLIK hrfs and the empirical and estimated TIITLIK chrfs for data set 1 .
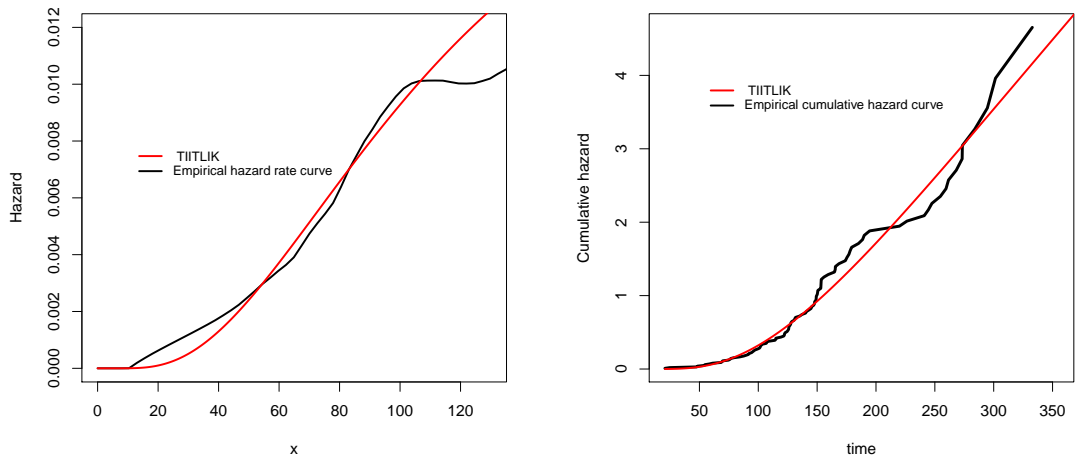

Figure 5. Pots of the empirical and estimated TIITLIK hrfs and the empirical and estimated TIITLIK chrfs for data set 2 .
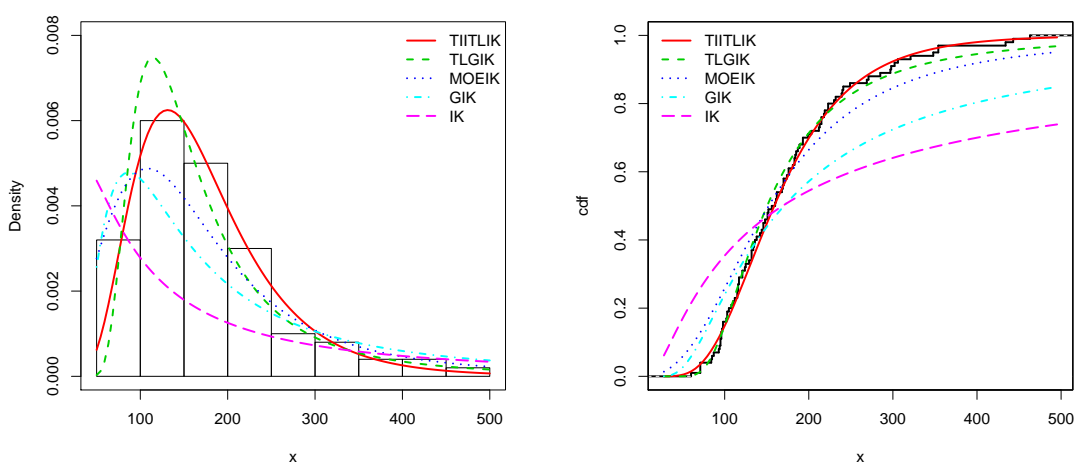

Figure 6. Plots of the estimated pdfs and cdfs for data set 1 . 

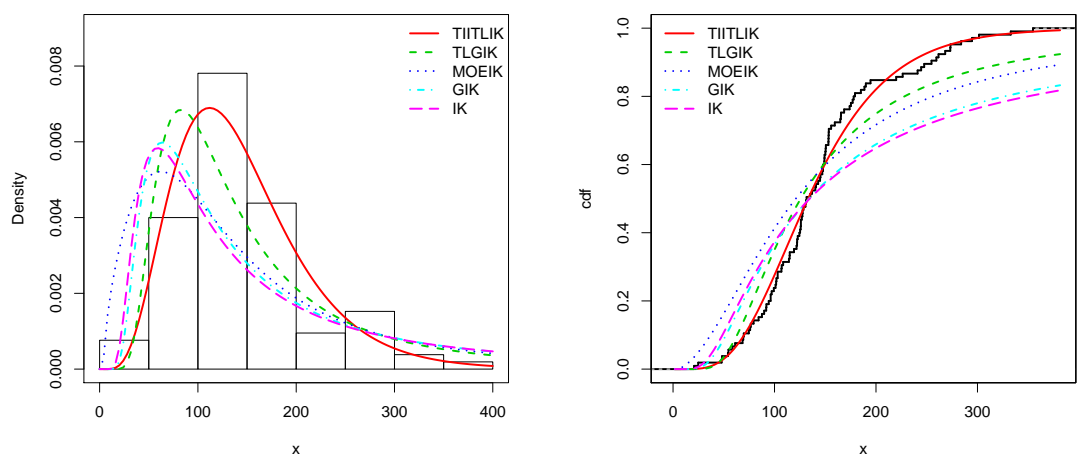

Figure 7. Plots of the estimated pdfs and cdfs for data set 2.

\section{On the MLEs of the TIITLIK Model}

Here, we discuss the definitions and the convergence properties of the MLEs of the TIITLIK model used in Section 3.

\subsection{Definition and Properties}

Let $x_{1}, \ldots, x_{n}$ be $n$ positive real numbers, all independent realisations of $X$. Then, the log-likelihood function for $\Theta=(\alpha, a, b, \lambda)$ is given by

$$
\begin{aligned}
\ell(\Theta) & =\sum_{i=1}^{n} \log \left[f\left(x_{i} ; \alpha, a, b, \lambda\right)\right]=n \log (2)+n \log (\alpha)+n \log (a)+n \log (b)-(a+1) \sum_{i=1}^{n} \log \left(1+x_{i}\right) \\
& +(2 b-1) \sum_{i=1}^{n} \log \left[1-\left(1+x_{i}\right)^{-a}\right]+\sum_{i=1}^{n} \log \left\{1+\lambda-2 \lambda\left[1-\left(1+x_{i}\right)^{-a}\right]^{b}\right\} \\
& +\sum_{i=1}^{n} \log \left\{1+\lambda-\lambda\left[1-\left(1+x_{i}\right)^{-a}\right]^{b}\right\} \\
& +(\alpha-1) \sum_{i=1}^{n} \log \left\{1-\left[1-\left(1+x_{i}\right)^{-a}\right]^{2 b}\left\{1+\lambda-\lambda\left[1-\left(1+x_{i}\right)^{-a}\right]^{b}\right\}^{2}\right\} .
\end{aligned}
$$

The vector of the MLEs of $\Theta$, say $\hat{\Theta}=(\hat{\alpha}, \hat{a}, \hat{b}, \hat{\lambda})$, are defined as the arguments of the maxima of $\ell(\Theta)$ according to $\Theta$. Thus, for the TIITLIK model, $\hat{\alpha}, \hat{a}, \hat{b}$ and $\hat{\lambda}$ can be determined as the simultaneous solutions of the following non-linear equations: $\partial \ell(\Theta) / \partial \alpha=0, \partial \ell(\Theta) / \partial a=0, \partial \ell(\Theta) / \partial b=0$ and $\partial \ell(\Theta) / \partial \lambda=0$, according to the parameters. Here, these partial derivatives can be expressed as follows:

$$
\begin{aligned}
& \frac{\partial \ell(\Theta)}{\partial \alpha}=\frac{n}{\alpha}+\sum_{i=1}^{n} \log \left\{1-\left[1-\left(1+x_{i}\right)^{-a}\right]^{2 b}\left\{1+\lambda-\lambda\left[1-\left(1+x_{i}\right)^{-a}\right]^{b}\right\}^{2}\right\}, \\
\frac{\partial \ell(\Theta)}{\partial a}= & \frac{n}{a}-\sum_{i=1}^{n} \log \left(1+x_{i}\right) \\
+ & (2 b-1) \sum_{i=1}^{n} \frac{\left(1+x_{i}\right)^{-a} \log \left(1+x_{i}\right)}{1-\left(1+x_{i}\right)^{-a}}-2 b \lambda \sum_{i=1}^{n} \frac{\left(1+x_{i}\right)^{-a} \log \left(1+x_{i}\right)\left[1-\left(1+x_{i}\right)^{-a}\right]^{b-1}}{1+\lambda-2 \lambda\left[1-\left(1+x_{i}\right)^{-a}\right]^{b}} \\
& -b \lambda \sum_{i=1}^{n} \frac{\left(1+x_{i}\right)^{-a} \log \left(1+x_{i}\right)\left[1-\left(1+x_{i}\right)^{-a}\right]^{b-1}}{1+\lambda-\lambda\left[1-\left(1+x_{i}\right)^{-a}\right]^{b}} \\
+ & 2 b(\alpha-1) \sum_{i=1}^{n} \frac{\left(1+x_{i}\right)^{-a} \log \left(1+x_{i}\right)\left[1-\left(1+x_{i}\right)^{-a}\right]^{2 b-1}\left\{1+\lambda-\lambda\left[1-\left(1+x_{i}\right)^{-a}\right]^{b}\right\}}{1-\left[1-\left(1+x_{i}\right)^{-a}\right]^{2 b}\left\{1+\lambda-\lambda\left[1-\left(1+x_{i}\right)^{-a}\right]^{b}\right\}^{2}} \times \\
& {\left[\lambda\left[1-\left(1+x_{i}\right)^{-a}\right]^{b}-\left\{1+\lambda-\lambda\left[1-\left(1+x_{i}\right)^{-a}\right]^{b}\right\}\right], }
\end{aligned}
$$




$$
\begin{aligned}
\frac{\partial \ell(\Theta)}{\partial b} & =\frac{n}{b}+2 \sum_{i=1}^{n} \log \left[1-\left(1+x_{i}\right)^{-a}\right]-2 \lambda \sum_{i=1}^{n} \frac{\left[1-\left(1+x_{i}\right)^{-a}\right]^{b} \log \left[1-\left(1+x_{i}\right)^{-a}\right]}{1+\lambda-2 \lambda\left[1-\left(1+x_{i}\right)^{-a}\right]^{b}} \\
& -\lambda \sum_{i=1}^{n} \frac{\left[1-\left(1+x_{i}\right)^{-a}\right]^{b} \log \left[1-\left(1+x_{i}\right)^{-a}\right]}{1+\lambda-\lambda\left[1-\left(1+x_{i}\right)^{-a}\right]^{b}} \\
& +2(\alpha-1) \sum_{i=1}^{n} \frac{\left[1-\left(1+x_{i}\right)^{-a}\right]^{2 b} \log \left[1-\left(1+x_{i}\right)^{-a}\right]\left\{1+\lambda-\lambda\left[1-\left(1+x_{i}\right)^{-a}\right]^{b}\right\}}{1-\left[1-\left(1+x_{i}\right)^{-a}\right]^{2 b}\left\{1+\lambda-\lambda\left[1-\left(1+x_{i}\right)^{-a}\right]^{b}\right\}^{2}} \times \\
& {\left[\lambda\left[1-\left(1+x_{i}\right)^{-a}\right]^{b}-\left\{1+\lambda-\lambda\left[1-\left(1+x_{i}\right)^{-a}\right]^{b}\right\}\right] }
\end{aligned}
$$

and

$$
\begin{aligned}
\frac{\partial \ell(\Theta)}{\partial \lambda} & =\sum_{i=1}^{n} \frac{1-2\left[1-\left(1+x_{i}\right)^{-a}\right]^{b}}{1+\lambda-2 \lambda\left[1-\left(1+x_{i}\right)^{-a}\right]^{b}}+\sum_{i=1}^{n} \frac{1-\left[1-\left(1+x_{i}\right)^{-a}\right]^{b}}{1+\lambda-\lambda\left[1-\left(1+x_{i}\right)^{-a}\right]^{b}} \\
& -2(\alpha-1) \sum_{i=1}^{n} \frac{\left[1-\left(1+x_{i}\right)^{-a}\right]^{2 b}\left\{1-\left[1-\left(1+x_{i}\right)^{-a}\right]^{b}\right\}\left\{1+\lambda-\lambda\left[1-\left(1+x_{i}\right)^{-a}\right]^{b}\right\}}{1-\left[1-\left(1+x_{i}\right)^{-a}\right]^{2 b}\left\{1+\lambda-\lambda\left[1-\left(1+x_{i}\right)^{-a}\right]^{b}\right\}^{2}} .
\end{aligned}
$$

One might notice that, thanks to (3), we have the following relation between the MLEs:

$$
\hat{\alpha}=\left[-\frac{1}{n} \sum_{i=1}^{n} \log \left\{1-\left[1-\left(1+x_{i}\right)^{-\hat{a}}\right]^{2 \hat{b}}\left\{1+\hat{\lambda}-\hat{\lambda}\left[1-\left(1+x_{i}\right)^{-\hat{a}}\right]^{\hat{b}}\right\}^{2}\right\}\right]^{-1} .
$$

Clearly, we have no analytical expression for the MLEs. However, nonlinear optimisation methods, such as the quasi-Newton algorithm, can be used to have a precise numerical evaluation. Under some regularity assumptions, when $n$ is large, the sub adjacent distribution of $\hat{\Theta}$ is close to a multivariate normal distribution $\mathcal{N}_{4}\left(0_{4}, J(\hat{\Theta})^{-1}\right)$, where $J(\hat{\Theta})=\left.\left\{-\partial^{2} \ell(\Theta) / \partial r \partial s\right\}_{(r, s) \in\{\alpha, a, b, \lambda\}^{2}}\right|_{\Theta=\hat{\Theta}}$ (the $4 \times 4$ observed information matrix evaluated at $\hat{\Theta})$. Here, for the sake of space, we omit its expression.

\subsection{Simulation}

Next, in the context of the TIITLIK model, we came up with a numerical study to study the behaviour of the MLEs and the behaviour of estimated survival and hazard rate functions defined with these estimates. We generated $N=1000$ random samples of size $n=30,50$ and 100 from $X$. Five sets of the parameters were assigned. The MLEs and root mean square errors (RMSEs) of $a, b, \alpha$ and $\lambda$ were determined. Then, the estimates of sf and hrf at points $x_{0}$ were also evaluated. We adopted the notations: $\hat{R}\left(x_{0}\right)=R\left(x_{0}, \hat{\alpha}, \hat{a}, \hat{b}, \hat{\lambda}\right)$ and $\hat{h}\left(x_{0}\right)=h\left(x_{0}, \hat{\alpha}, \hat{a}, \hat{b}, \hat{\lambda}\right)$ (we remind you that $\hat{\alpha}, \hat{a}, \hat{b}$ and $\hat{\lambda}$ denote the MLEs of $\alpha, a, b$ and $\lambda$, respectively). The numerical results are documented in Tables 6-10. Table 6 considers the set of parameters: $(a=0.5, b=2, \alpha=2$ and $\lambda=0.5)$; Table 7 considers: $(a=0.5$, $b=2, \alpha=2$ and $\lambda=0.2)$; Table 8 considers: $(a=0.5, b=2, \alpha=3$ and $\lambda=0.5)$; Table 9 considers: ( $a=0.5, b=2, \alpha=3$ and $\lambda=0.2)$; and finally, Table 9 considers: $(a=1.2, b=3, \alpha=2$ and $\lambda=0.5)$. 
Table 6. MLEs, RMSEs and estimated sf and hrf of the TIITLIK distribution $(a=0.5, b=2, \alpha=2$ and $\lambda=0.5)$.

\begin{tabular}{|c|c|c|c|c|c|c|c|c|c|}
\hline & Parameters & ML & RMSE & & $\hat{R}\left(x_{0}\right)$ & & & $\hat{h}\left(x_{0}\right)$ & \\
\hline$n$ & & & & $x_{0}=3$ & $x_{0}=5$ & $x_{0}=7$ & $x_{0}=3$ & $x_{0}=5$ & $x_{0}=7$ \\
\hline \multirow{4}{*}{30} & $a$ & 0.800 & 0.550 & \multirow{4}{*}{0.736} & \multirow{4}{*}{0.515} & \multirow{4}{*}{0.371} & \multirow{4}{*}{0.301} & \multirow{4}{*}{0.264} & \multirow{4}{*}{0.221} \\
\hline & $b$ & 2.831 & 1.928 & & & & & & \\
\hline & $\alpha$ & 2.518 & 2.037 & & & & & & \\
\hline & $\lambda$ & 0.078 & 0.616 & & & & & & \\
\hline \multirow{4}{*}{50} & $a$ & 0.770 & 0.460 & \multirow{4}{*}{0.743} & \multirow{4}{*}{0.540} & \multirow{4}{*}{0.406} & \multirow{4}{*}{0.285} & \multirow{4}{*}{0.240} & \multirow{4}{*}{0.197} \\
\hline & $b$ & 2.610 & 1.586 & & & & & & \\
\hline & $\alpha$ & 2.172 & 1.590 & & & & & & \\
\hline & $\lambda$ & 0.113 & 0.570 & & & & & & \\
\hline \multirow{4}{*}{100} & $a$ & 0.763 & 0.358 & \multirow{4}{*}{0.750} & \multirow{4}{*}{0.565} & \multirow{4}{*}{0.442} & \multirow{4}{*}{0.253} & \multirow{4}{*}{0.205} & \multirow{4}{*}{0.165} \\
\hline & $b$ & 2.451 & 0.910 & & & & & & \\
\hline & $\alpha$ & 1.716 & 1.007 & & & & & & \\
\hline & $\lambda$ & 0.184 & 0.469 & & & & & & \\
\hline
\end{tabular}

Table 7. MLEs, RMSEs, and estimated sf and hrf of the TIITLIK distribution $(a=0.5, b=2, \alpha=2$ and $\lambda=0.2)$

\begin{tabular}{|c|c|c|c|c|c|c|c|c|c|}
\hline & Parameters & ML & RMSE & & $\hat{R}\left(x_{0}\right)$ & & & $\hat{h}\left(x_{0}\right)$ & \\
\hline$n$ & & & & $x_{0}=3$ & $x_{0}=5$ & $x_{0}=7$ & $x_{0}=3$ & $x_{0}=5$ & $x_{0}=7$ \\
\hline \multirow{4}{*}{30} & $a$ & 0.765 & 0.494 & \multirow{4}{*}{0.816} & \multirow{4}{*}{0.622} & \multirow{4}{*}{0.478} & \multirow{4}{*}{0.262} & \multirow{4}{*}{0.240} & \multirow{4}{*}{0.204} \\
\hline & $b$ & 3.058 & 2.314 & & & & & & \\
\hline & $\alpha$ & 2.436 & 2.519 & & & & & & \\
\hline & $\lambda$ & 0.053 & 0.466 & & & & & & \\
\hline \multirow{4}{*}{50} & $a$ & 0.641 & 0.283 & \multirow{4}{*}{0.823} & \multirow{4}{*}{0.665} & \multirow{4}{*}{0.546} & \multirow{4}{*}{0.253} & \multirow{4}{*}{0.218} & \multirow{4}{*}{0.176} \\
\hline & $b$ & 2.374 & 1.232 & & & & & & \\
\hline & $\alpha$ & 2.088 & 1.538 & & & & & & \\
\hline & $\lambda$ & 0.072 & 0.435 & & & & & & \\
\hline \multirow{4}{*}{100} & $a$ & 0.652 & 0.265 & \multirow{4}{*}{0.824} & \multirow{4}{*}{0.671} & \multirow{4}{*}{0.558} & \multirow{4}{*}{0.240} & \multirow{4}{*}{0.196} & \multirow{4}{*}{0.158} \\
\hline & $b$ & 2.325 & 0.872 & & & & & & \\
\hline & $\alpha$ & 1.867 & 1.165 & & & & & & \\
\hline & $\lambda$ & 0.071 & 0.390 & & & & & & \\
\hline
\end{tabular}

Table 8. MLEs, RMSEs and estimated sf and hrf of the TIITLIK distribution $(a=0.5, b=2, \alpha=3$ and $\lambda=0.5)$.

\begin{tabular}{|c|c|c|c|c|c|c|c|c|c|}
\hline & Parameters & ML & RMSE & & $\hat{R}\left(x_{0}\right)$ & & & $\hat{h}\left(x_{0}\right)$ & \\
\hline$n$ & & & & $x_{0}=3$ & $x_{0}=5$ & $x_{0}=7$ & $x_{0}=3$ & $x_{0}=5$ & $x_{0}=7$ \\
\hline \multirow{4}{*}{30} & $a$ & 0.879 & 0.644 & \multirow{4}{*}{0.591} & \multirow{4}{*}{0.324} & \multirow{4}{*}{0.190} & \multirow{4}{*}{0.423} & \multirow{4}{*}{0.372} & \multirow{4}{*}{0.309} \\
\hline & $b$ & 3.113 & 2.796 & & & & & & \\
\hline & $\alpha$ & 3.104 & 2.303 & & & & & & \\
\hline & $\lambda$ & 0.266 & 0.930 & & & & & & \\
\hline \multirow{4}{*}{50} & $a$ & 0.855 & 0.519 & \multirow{4}{*}{0.615} & \multirow{4}{*}{0.382} & \multirow{4}{*}{0.253} & \multirow{4}{*}{0.338} & \multirow{4}{*}{0.285} & \multirow{4}{*}{0.235} \\
\hline & $b$ & 2.523 & 1.207 & & & & & & \\
\hline & $\alpha$ & 2.584 & 1.668 & & & & & & \\
\hline & $\lambda$ & 0.065 & 0.595 & & & & & & \\
\hline \multirow{4}{*}{100} & $a$ & 0.836 & 0.443 & \multirow{4}{*}{0.651} & \multirow{4}{*}{0.426} & \multirow{4}{*}{0.294} & \multirow{4}{*}{0.314} & \multirow{4}{*}{0.265} & \multirow{4}{*}{0.218} \\
\hline & $b$ & 2.507 & 1.081 & & & & & & \\
\hline & $\alpha$ & 2.404 & 1.495 & & & & & & \\
\hline & $\lambda$ & 0.064 & 0.581 & & & & & & \\
\hline
\end{tabular}


Table 9. MLEs, RMSEs and estimated sf and hrf of the TIITLIK distribution $(a=0.5, b=2, \alpha=3$ and $\lambda=0.2)$.

\begin{tabular}{|c|c|c|c|c|c|c|c|c|c|}
\hline & Parameters & ML & RMSE & & $\hat{R}\left(x_{0}\right)$ & & & $\hat{h}\left(x_{0}\right)$ & \\
\hline$n$ & & & & $x_{0}=3$ & $x_{0}=5$ & $x_{0}=7$ & $x_{0}=3$ & $x_{0}=5$ & $x_{0}=7$ \\
\hline \multirow{4}{*}{30} & $a$ & 0.800 & 0.493 & \multirow{4}{*}{0.723} & \multirow{4}{*}{0.495} & \multirow{4}{*}{0.349} & \multirow{4}{*}{0.316} & \multirow{4}{*}{0.280} & \multirow{4}{*}{0.234} \\
\hline & $b$ & 2.808 & 1.775 & & & & & & \\
\hline & $\alpha$ & 2.737 & 1.983 & & & & & & \\
\hline & $\lambda$ & 0.044 & 0.482 & & & & & & \\
\hline \multirow{4}{*}{50} & $a$ & 0.767 & 0.446 & \multirow{4}{*}{0.728} & \multirow{4}{*}{0.513} & \multirow{4}{*}{0.374} & \multirow{4}{*}{0.313} & \multirow{4}{*}{0.268} & \multirow{4}{*}{0.221} \\
\hline & $b$ & 2.623 & 1.453 & & & & & & \\
\hline & $\alpha$ & 2.521 & 1.85 & & & & & & \\
\hline & $\lambda$ & 0.068 & 0.466 & & & & & & \\
\hline \multirow{4}{*}{100} & $a$ & 0.740 & 0.354 & \multirow{4}{*}{0.738} & \multirow{4}{*}{0.541} & \multirow{4}{*}{0.410} & \multirow{4}{*}{0.283} & \multirow{4}{*}{0.236} & \multirow{4}{*}{0.194} \\
\hline & $b$ & 2.353 & 0.966 & & & & & & \\
\hline & $\alpha$ & 2.320 & 1.496 & & & & & & \\
\hline & $\lambda$ & -0.00542 & 0.449 & & & & & & \\
\hline
\end{tabular}

Table 10. MLEs, RMSEs and estimated sf and hrf of the TIITLIK distribution $(a=1.2, b=3, \alpha=2$ and $\lambda=0.5)$.

\begin{tabular}{|c|c|c|c|c|c|c|c|c|c|}
\hline & Parameters & ML & RMSE & & $\hat{R}\left(x_{0}\right)$ & & & $\hat{h}\left(x_{0}\right)$ & \\
\hline$n$ & & & & $x_{0}=3$ & $x_{0}=5$ & $x_{0}=7$ & $x_{0}=3$ & $x_{0}=5$ & $x_{0}=7$ \\
\hline \multirow{4}{*}{30} & $a$ & 1.530 & 0.898 & \multirow{4}{*}{0.151} & \multirow{4}{*}{0.032} & \multirow{4}{*}{0.010} & \multirow{4}{*}{0.269} & \multirow{4}{*}{0.278} & \multirow{4}{*}{0.252} \\
\hline & $b$ & 4.257 & 2.831 & & & & & & \\
\hline & $\alpha$ & 2.261 & 1.682 & & & & & & \\
\hline & $\lambda$ & 0.705 & 0.958 & & & & & & \\
\hline \multirow{4}{*}{50} & $a$ & 1.504 & 0.778 & \multirow{4}{*}{0.191} & \multirow{4}{*}{0.052} & \multirow{4}{*}{0.020} & \multirow{4}{*}{0.265} & \multirow{4}{*}{0.257} & \multirow{4}{*}{0.229} \\
\hline & $b$ & 3.945 & 2.433 & & & & & & \\
\hline & $\alpha$ & 2.068 & 1.486 & & & & & & \\
\hline & $\lambda$ & 0.620 & 0.917 & & & & & & \\
\hline \multirow{4}{*}{100} & $a$ & 1.464 & 0.721 & \multirow{4}{*}{0.229} & \multirow{4}{*}{0.073} & \multirow{4}{*}{0.031} & \multirow{4}{*}{0.262} & \multirow{4}{*}{0.252} & \multirow{4}{*}{0.223} \\
\hline & $b$ & 3.633 & 1.794 & & & & & & \\
\hline & $\alpha$ & 2.057 & 1.204 & & & & & & \\
\hline & $\lambda$ & 0.481 & 0.439 & & & & & & \\
\hline
\end{tabular}

From these numerical results, we observe that the RMSEs decrease as $n$ increases in all situations. The estimated sfs decrease when $x_{0}$ increases in all situations. The estimated hrfs decrease when $x_{0}$ increases in all situations. The estimated sfs increase as $n$ increases in all situations. The estimated hrfs decrease as $n$ increases in all situations.

\section{Mathematical Properties}

After the practical aspect, this section is devoted to the main mathematical properties of the TIITLIK distribution. Hereafter, we consider a random variable $X$ following the TIITLIK distribution; i.e., having the cdf given by (1) and the pdf given by (2).

\subsection{Asymptotic Results and Critical Points}

Some asymptotic results and critical points of the main functions of the TIITLIK distribution are presented below. When $x \rightarrow 0$, by using standard equivalence formulas, we get

$$
F(x ; \alpha, a, b, \lambda) \sim \alpha(1+\lambda)^{2} a^{2 b} x^{2 b}, \quad f(x ; \alpha, a, b, \lambda) \sim 2 b \alpha(1+\lambda)^{2} a^{2 b} x^{2 b-1}
$$


and

$$
h(x ; \alpha, a, b, \lambda) \sim 2 b \alpha(1+\lambda)^{2} a^{2 b} x^{2 b-1} .
$$

Hence, the limits of these functions mainly depend on $b$. When $b \in(0,1 / 2)$, we get $f(x ; \alpha, a, b, \lambda) \rightarrow$ $+\infty$; when $b=1 / 2$, we obtain $f(x ; \alpha, a, b, \lambda) \rightarrow \alpha(1+\lambda)^{2} a$; and when $b>1 / 2$, we get $f(x ; \alpha, a, b, \lambda) \rightarrow$ 0 . Similarly, when $b \in(0,1 / 2)$, we get $h(x ; \alpha, a, b, \lambda) \rightarrow+\infty$; when $b=1 / 2$, we obtain $h(x ; \alpha, a, b, \lambda) \rightarrow$ $\alpha(1+\lambda)^{2} a$; and when $b>1 / 2$, we get $h(x ; \alpha, a, b, \lambda) \rightarrow 0$.

Now, when $x \rightarrow+\infty$, we get

$$
F(x ; \alpha, a, b, \lambda) \sim 1-2^{\alpha} b^{\alpha} x^{-a \alpha}, \quad f(x ; \alpha, a, b, \lambda) \sim 2^{\alpha} b^{\alpha} a \alpha x^{-a \alpha-1}
$$

and

$$
h(x ; \alpha, a, b, \lambda) \sim a \alpha x^{-1} .
$$

Hence, in all cases, we have $f(x ; \alpha, a, b, \lambda) \rightarrow 0$ and $h(x ; \alpha, a, b, \lambda) \rightarrow 0$. We would like to mention that $\lambda$ plays no role for these asymptotes.

Let us now study the critical points of $f(x ; \alpha, a, b, \lambda)$ and $h(x ; \alpha, a, b, \lambda)$. The critical point(s) of $f(x ; \alpha, a, b, \lambda)$ is(are) given by the solution(s) of the following equation: $\{\log [f(x ; \alpha, a, b, \lambda)]\}^{\prime}=0$ (the derivative is according to $x$ ), where

$$
\begin{aligned}
& \log [f(x ; \alpha, a, b, \lambda)]=\log (2)+\log (\alpha)+\log (a)+\log (b)-(a+1) \log (1+x) \\
& +(2 b-1) \log \left[1-(1+x)^{-a}\right]+\log \left\{1+\lambda-2 \lambda\left[1-(1+x)^{-a}\right]^{b}\right\} \\
& +\log \left\{1+\lambda-\lambda\left[1-(1+x)^{-a}\right]^{b}\right\} \\
& +(\alpha-1) \log \left\{1-\left[1-(1+x)^{-a}\right]^{2 b}\left\{1+\lambda-\lambda\left[1-(1+x)^{-a}\right]^{b}\right\}^{2}\right\}
\end{aligned}
$$

Then, a critical point $x_{0}$ of $f(x ; \alpha, a, b, \lambda)$ satisfies the following equation:

$$
\begin{aligned}
& -(a+1) \frac{1}{1+x_{0}}+a(2 b-1) \frac{\left(1+x_{0}\right)^{-a-1}}{1-\left(1+x_{0}\right)^{-a}}-2 a b \lambda \frac{\left(1+x_{0}\right)^{-a-1}\left[1-\left(1+x_{0}\right)^{-a}\right]^{b-1}}{1+\lambda-2 \lambda\left[1-\left(1+x_{0}\right)^{-a}\right]^{b}}+ \\
& -a b \lambda \frac{\left(1+x_{0}\right)^{-a-1}\left[1-\left(1+x_{0}\right)^{-a}\right]^{b-1}}{1+\lambda-\lambda\left[1-\left(1+x_{0}\right)^{-a}\right]^{b}} \\
& +2(\alpha-1) a b \frac{\left(1+x_{0}\right)^{-a-1}\left[1-\left(1+x_{0}\right)^{-a}\right]^{2 b-1}\left\{1+\lambda-\lambda\left[1-\left(1+x_{0}\right)^{-a}\right]^{b}\right\}}{1-\left[1-\left(1+x_{0}\right)^{-a}\right]^{2 b}\left\{1+\lambda-\lambda\left[1-\left(1+x_{0}\right)^{-a}\right]^{b}\right\}^{2}} \times \\
& {\left[\lambda\left[1-\left(1+x_{0}\right)^{-a}\right]^{b}-\left\{1+\lambda-\lambda\left[1-\left(1+x_{0}\right)^{-a}\right]^{b}\right\}\right]=0 .}
\end{aligned}
$$

The nature of $x_{0}$ can be determined by investigating the sign of $\left.\{\log [f(x ; \alpha, a, b, \lambda)]\}^{\prime \prime}\right|_{x=x_{0}}$. Due to the complexity of these equations, for given values of $\alpha, a, b$ and $\lambda$, one can make use of mathematical software to determine $x_{0}$ : R, Mathematica, Matlab, etc.

Similarly, the critical point(s) of $h(x ; \alpha, a, b, \lambda)$ is(are) given by the solution(s) of the following equation: $\{\log [h(x ; \alpha, a, b, \lambda)]\}^{\prime}=0$. If such a critical point is denoted by $x_{*}$, it satisfies the following equation: 


$$
\begin{aligned}
& -(a+1) \frac{1}{1+x_{*}}+a(2 b-1) \frac{\left(1+x_{*}\right)^{-a-1}}{1-\left(1+x_{*}\right)^{-a}}-2 a b \lambda \frac{\left(1+x_{*}\right)^{-a-1}\left[1-\left(1+x_{*}\right)^{-a}\right]^{b-1}}{1+\lambda-2 \lambda\left[1-\left(1+x_{*}\right)^{-a}\right]^{b}}+ \\
& -a b \lambda \frac{\left(1+x_{*}\right)^{-a-1}\left[1-\left(1+x_{*}\right)^{-a}\right]^{b-1}}{1+\lambda-\lambda\left[1-\left(1+x_{*}\right)^{-a}\right]^{b}} \\
& -2 a b \frac{\left(1+x_{*}\right)^{-a-1}\left[1-\left(1+x_{*}\right)^{-a}\right]^{2 b-1}\left\{1+\lambda-\lambda\left[1-\left(1+x_{*}\right)^{-a}\right]^{b}\right\}}{1-\left[1-\left(1+x_{*}\right)^{-a}\right]^{2 b}\left\{1+\lambda-\lambda\left[1-\left(1+x_{*}\right)^{-a}\right]^{b}\right\}^{2}} \times \\
& {\left[\lambda\left[1-\left(1+x_{*}\right)^{-a}\right]^{b}-\left\{1+\lambda-\lambda\left[1-\left(1+x_{*}\right)^{-a}\right]^{b}\right\}\right]=0 .}
\end{aligned}
$$

Again, the nature of $x_{*}$ can be determined by investigating the sign of $\left.\{\log [h(x ; \alpha, a, b, \lambda)]\}^{\prime \prime}\right|_{x=x_{*}}$.

\subsection{Quantile Function}

The quantile function (qf) of $X$, say $Q(y ; \alpha, a, b, \lambda)$, is characterised by the following equation: $Q(F(y ; \alpha, a, b, \lambda) ; \alpha, a, b, \lambda)=F(Q(y ; \alpha, a, b, \lambda) ; \alpha, a, b, \lambda)=y, y \in(0,1)$. After some algebra, we obtain

$$
Q(y ; \alpha, a, b, \lambda)=\left\{1-\left[\frac{1+\lambda-\sqrt{1+2\left[1-2 \sqrt{1-(1-y)^{1 / \alpha}}\right] \lambda+\lambda^{2}}}{2 \lambda}\right]^{1 / b}-1, \quad y \in(0,1) .\right.
$$

Several quantities can be defined via the quantile function. For instance, the second quartile (median) is given by $M=Q(1 / 2 ; \alpha, a, b, \lambda)$ and the inter-quantile range can be expressed as $I Q R=$ $Q(3 / 4 ; \alpha, a, b, \lambda)-Q(1 / 4 ; \alpha, a, b, \lambda)$.

Also, one can generate values from $X$ by using the following result. Let $x_{U}=Q(U ; \alpha, a, b, \lambda)$, where $U$ denotes a random variable following the uniform distribution $\mathcal{U}(0,1)$. Then, $x_{U}$ follows the TIITLIK distribution. This result was used in Section 4.2.

\subsection{Bowley Skewness and Moors Kurtosis}

The Bowley skewness and Moors kurtosis of $X$ are defined by, respectively,

$$
S=\frac{Q(6 / 8 ; \alpha, a, b, \lambda)+Q(2 / 8 ; \alpha, a, b, \lambda)-2 Q(4 / 8 ; \alpha, a, b, \lambda)}{I Q R}
$$

and

$$
K=\frac{Q(7 / 8 ; \alpha, a, b, \lambda)-Q(5 / 8 ; \alpha, a, b, \lambda)+Q(3 / 8 ; \alpha, a, b, \lambda)-Q(1 / 8 ; \alpha, a, b, \lambda)}{I Q R} .
$$

Here, $S$ is a measure of the asymmetry of the TIITLIK distribution and $K$ is a measure of whether the TIITLIK distributions are heavy-tailed or light-tailed (relatively to a normal distribution). These measures have the advantage of always being well-defined from the mathematical point of view, contrary to other skewness and kurtosis measures (based on moments, for instance). More detail can be found in [22] for the Bowley skewness and [23] for the Moors kurtosis.

The plots of $S$ and $K$ are presented in Figures 8-11 for $\alpha, a \in(1,5), b=5$ and varying values for $\lambda$; i.e., $\lambda \in\{-0.3,-0.8,0.3,0.8\}$. The impacts of the values of the parameters on $S$ and $K$ are significant; we can see that $S$ can be both positive and negative, with complex variations. Similarly, $K$ either increases or decreases, depending on the values of the parameters. 

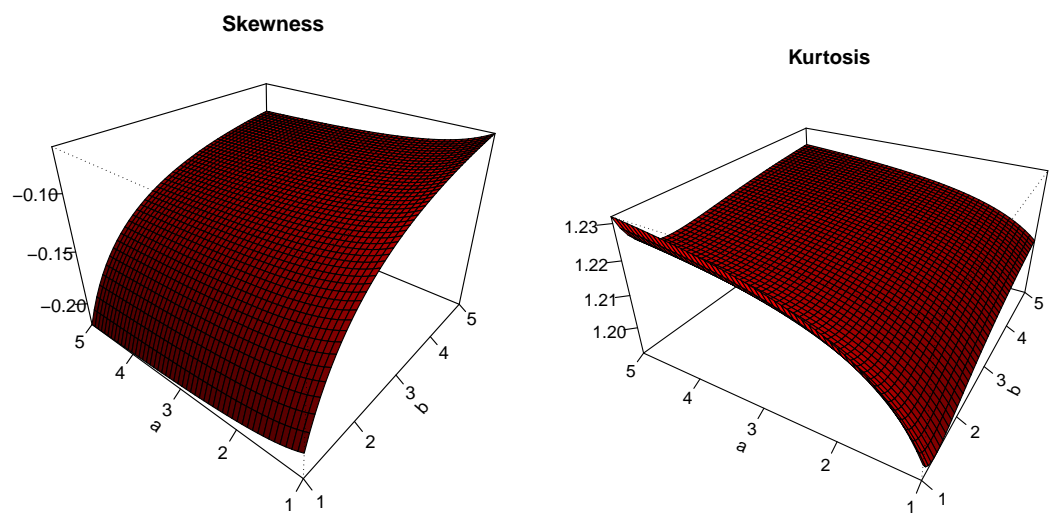

Figure 8. Plots for Bowley skewness and Moors kurtosis for $\alpha, a \in(1,5), b=5$ and $\lambda=-0.3$.
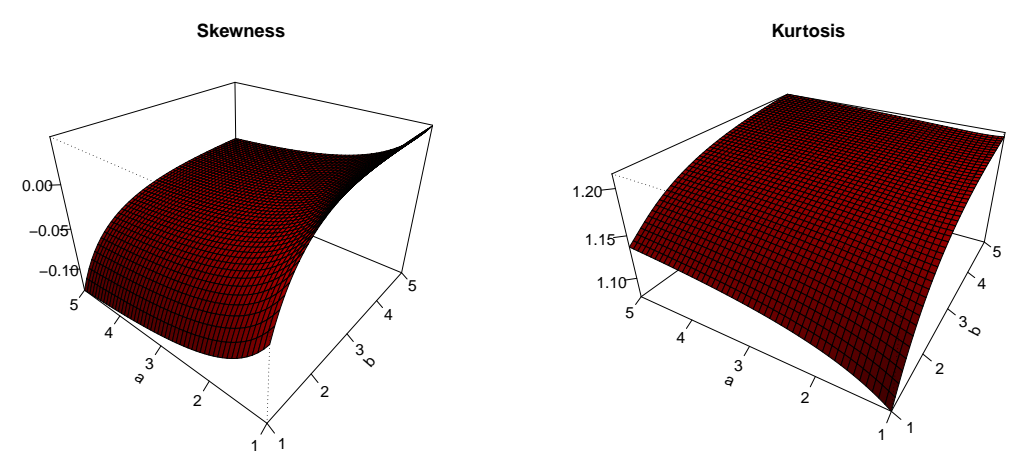

Figure 9. Plots for Bowley skewness and Moors kurtosis for $\alpha, a \in(1,5), b=5$ and $\lambda=-0.8$.
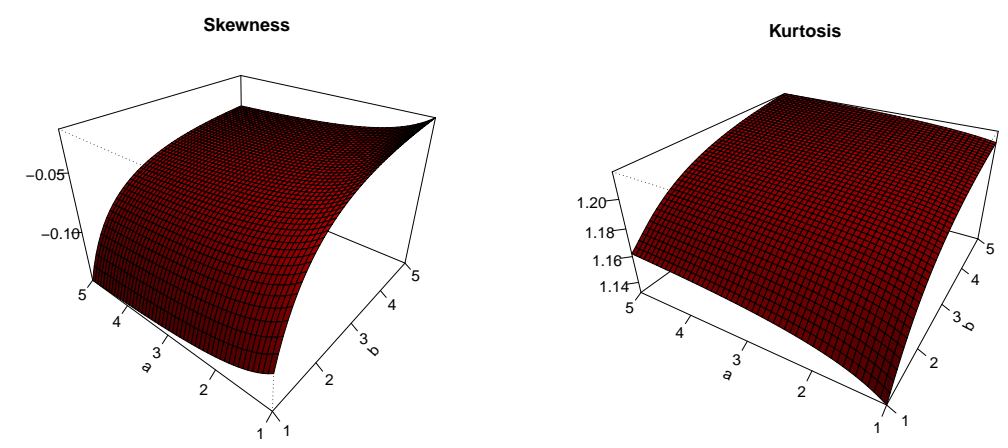

Figure 10. Plots for Bowley skewness and Moors kurtosis for $\alpha, a \in(1,5), b=5$ and $\lambda=0.3$.
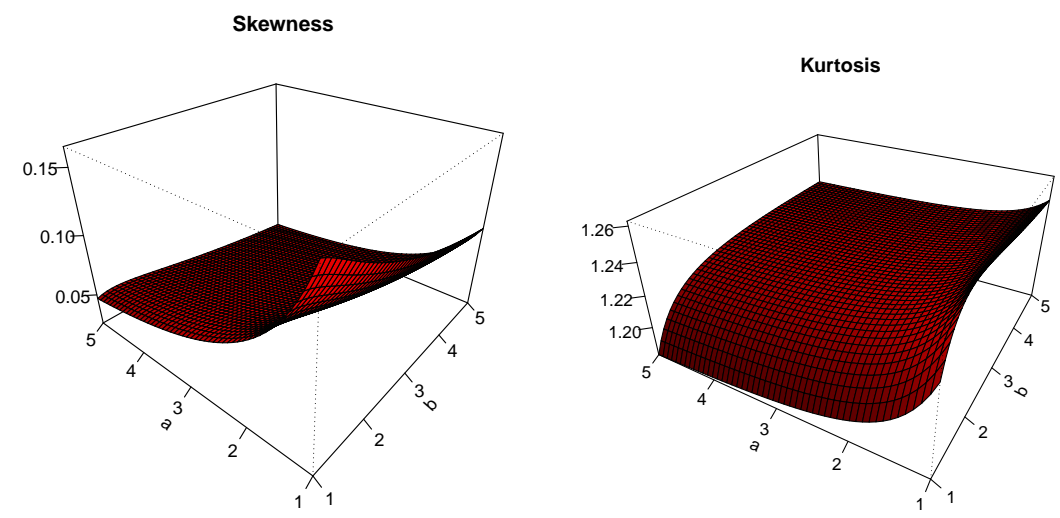

Figure 11. Plots for Bowley skewness and Moors kurtosis for $\alpha, a \in(1,5), b=5$ and $\lambda=0.8$. 


\subsection{Mixture Representations}

The three following results present a mixture of representations of some functions of the TIITLIK distribution in terms of Lomax distribution functions. In the proofs, for the sake of simplicity, we adopted the notation introduced in Section 1.

Proposition 1. The following is a mixture representation for $F(x ; \alpha, a, b, \lambda)$ :

$$
F(x ; \alpha, a, b, \lambda)=1-\sum_{k=0}^{+\infty} \sum_{\ell=0}^{2 k} \sum_{m=0}^{+\infty} \varphi_{k, \ell, m} S_{m}(x ; a),
$$

where $\varphi_{k, \ell, m}=\left(\begin{array}{c}\alpha \\ k\end{array}\right)\left(\begin{array}{c}2 k \\ \ell\end{array}\right)\left(\begin{array}{c}b(\ell+2 k) \\ m\end{array}\right)(-1)^{k+\ell+m}(1+\lambda)^{2 k-\ell} \lambda^{\ell},\left(\right.$ with $\left.\left(\begin{array}{l}v \\ u\end{array}\right)=v(v-1) \ldots(v-u+1) / u !\right)$ and $S_{m}(x ; a)$ denote the sf of the Lomax distribution with parameters am and 1 ; i.e., $S_{m}(x)=(1+x)^{-a m}$.

Proof. Since $G(x ; a, b, \lambda) \in(0,1)$ (excluding the limit points), the generalised binomial formula gives

$$
F(x ; \alpha, a, b, \lambda)=1-\sum_{k=0}^{+\infty}\left(\begin{array}{l}
\alpha \\
k
\end{array}\right)(-1)^{k}[G(x ; a, b, \lambda)]^{2 k} .
$$

Furthermore, it follows from the standard binomial formula that

$$
\begin{aligned}
{[G(x ; a, b, \lambda)]^{2 k} } & =\left[G_{*}(x ; a, b)\right]^{2 k}\left[1+\lambda-\lambda G_{*}(x ; a, b)\right]^{2 k} \\
& =\sum_{\ell=0}^{2 k}\left(\begin{array}{c}
2 k \\
\ell
\end{array}\right)(1+\lambda)^{2 k-\ell}(-\lambda)^{\ell}\left[G_{*}(x ; a, b)\right]^{\ell+2 k} .
\end{aligned}
$$

Since $(1+x)^{-a} \in(0,1)$, by applying again the generalised binomial formula, we have

$$
\left[G_{*}(x ; a, b)\right]^{\ell+2 k}=\left[1-(1+x)^{-a}\right]^{b(\ell+2 k)}=\sum_{m=0}^{+\infty}\left(\begin{array}{c}
b(\ell+2 k) \\
m
\end{array}\right)(-1)^{m} S_{m}(x ; a) .
$$

We end the proof by combining the above equalities together.

Proposition 2. We have the following mixture representation for $f(x ; \alpha, a, b, \lambda)$ :

$$
f(x ; \alpha, a, b, \lambda)=\sum_{k=0}^{+\infty} \sum_{\ell=0}^{2 k} \sum_{m=1}^{+\infty} \varphi_{k, \ell, m} f_{m}(x ; a),
$$

where $\varphi_{k, \ell, m}$ is defined as in Proposition 1 and $f_{m}(x ; a)$ denotes the $p d f$ of the Lomax distribution with parameters am and 1 , i.e., $f_{m}(x ; a)=a m(1+x)^{-a m-1}$.

Proof. The result follows from differentiation of the mixture expansion of $F(x ; \alpha, a, b, \lambda)$ established in Proposition 1.

Owing to Proposition 2, we can exploit the properties of the Lomax distribution to derive new properties for the TIITLIK distribution. This methodology will be used in the next subsections.

Proposition 3. Let $\xi$ be a positive integer. Then, we have the following mixture representation:

$$
f(x ; \alpha, a, b, \lambda) F(x ; \alpha, a, b, \lambda)^{\xi}=\sum_{k=0}^{\xi+1} \sum_{\ell=0}^{+\infty} \sum_{m=0}^{2 \ell} \sum_{q=1}^{+\infty} \phi_{k, \ell, m, q}^{(\xi)} f_{q}(x ; a),
$$


where $\phi_{k, \ell, m, q}^{(\xi)}=\left(\begin{array}{c}\xi+1 \\ k\end{array}\right)\left(\begin{array}{c}\alpha k \\ \ell\end{array}\right)\left(\begin{array}{c}2 \ell \\ m\end{array}\right)\left(\begin{array}{c}b(m+2 k) \\ q\end{array}\right)(-1)^{k+\ell+m+q+1}(\xi+1)^{-1}(1+\lambda)^{2 \ell-m} \lambda^{m}$ and $f_{q}(x ; a)$ denotes the pdf of the Lomax distribution with parameters aq and 1.

Proof. We will investigate a mixture representation for $F(x ; \alpha, a, b, \lambda)^{\xi+1}$ first, then consider the relation: $\left[F(x ; \alpha, a, b, \lambda)^{\xi+1}\right]^{\prime}=(\xi+1) f(x ; \alpha, a, b, \lambda) F(x ; \alpha, a, b, \lambda)^{\xi}$. Thus, it follows from the standard binomial formula that

$$
F(x ; \alpha, a, b, \lambda)^{\xi+1}=\left\{1-\left\{1-[G(x ; a, b, \lambda)]^{2}\right\}^{\alpha}\right\}^{\xi+1}=\sum_{k=0}^{\xi+1}\left(\begin{array}{c}
\xi+1 \\
k
\end{array}\right)(-1)^{k}\left\{1-[G(x ; a, b, \lambda)]^{2}\right\}^{\alpha k} .
$$

Since $G(x ; a, b, \lambda) \in(0,1)$, the generalised binomial formula gives

$$
\left\{1-[G(x ; a, b, \lambda)]^{2}\right\}^{\alpha k}=\sum_{\ell=0}^{+\infty}\left(\begin{array}{c}
\alpha k \\
\ell
\end{array}\right)(-1)^{\ell}[G(x ; a, b, \lambda)]^{2 \ell} .
$$

Then, we should proceed as in the proof of Proposition 1. The standard binomial formula gives

$$
[G(x ; a, b, \lambda)]^{2 \ell}=\sum_{m=0}^{2 \ell}\left(\begin{array}{c}
2 \ell \\
m
\end{array}\right)(1+\lambda)^{2 \ell-m}(-\lambda)^{m}\left[G_{*}(x ; a, b)\right]^{m+2 \ell}
$$

and, by the generalised binomial formula using $(1+x)^{-a} \in(0,1)$, we get

$$
\left[G_{*}(x ; a, b)\right]^{m+2 k}=\sum_{q=0}^{+\infty}\left(\begin{array}{c}
b(m+2 k) \\
q
\end{array}\right)(-1)^{q} S_{q}(x ; a) .
$$

By putting the above equalities together, we get

$$
F(x ; \alpha, a, b, \lambda)^{\xi+1}=\sum_{k=0}^{\xi+1} \sum_{\ell=0}^{+\infty} \sum_{m=0}^{2 \ell} \sum_{q=0}^{+\infty} \psi_{k, \ell, m, q}^{(\xi)} S_{q}(x ; a),
$$

where $\psi_{k, \ell, m, q}^{(\xi)}=\left(\begin{array}{c}\xi+1 \\ k\end{array}\right)\left(\begin{array}{c}\alpha k \\ \ell\end{array}\right)\left(\begin{array}{c}2 \ell \\ m\end{array}\right)\left(\begin{array}{c}b(m+2 k) \\ q\end{array}\right)(-1)^{k+\ell+m+q}(1+\lambda)^{2 \ell-m} \lambda^{m}$. Upon differentiation of this series expansion, we get

$$
f(x ; \alpha, a, b, \lambda) F(x ; \alpha, a, b, \lambda)^{\xi}=\sum_{k=0}^{\xi+1} \sum_{\ell=0}^{+\infty} \sum_{m=0}^{2 \ell} \sum_{q=1}^{+\infty} \phi_{k, \ell, m, q}^{(\xi)} f_{q}(x ; a),
$$

where $\phi_{k, \ell, m, q}^{(\mathcal{G})}=-(\xi+1)^{-1} \psi_{k, \ell, m, q^{\prime}}^{(\tilde{\xi})}$, which is the desired result.

\subsection{The Ordinary and Central Moments}

Let $r$ be a positive integer. Then, the $r$-th moment of $X$, i.e., $\mu_{r}^{\prime}=E\left(X^{r}\right)$, exists if, and only if, $a \alpha>r$. Under this assumption, we have

$$
\mu_{r}^{\prime}=\int_{0}^{+\infty} x^{r} f(x ; \alpha, a, b, \lambda) d x .
$$

For given values of $\alpha, a, b$ and $\lambda$, this integral can be evaluated by any mathematical software. Additionally, one can use Proposition 2 for a series expression, which is performed below. Let us introduce the gamma function defined by $\Gamma(s)=\int_{0}^{+\infty} t^{s} e^{-t} d t, s>0$. Then, by assuming that $a \min (\alpha, 1)>r$ and noticing that

$$
\int_{0}^{+\infty} x^{r} f_{m}(x ; a) d x=a m \frac{\Gamma(r+1) \Gamma(a m-r)}{\Gamma(a m+1)}=\frac{r !}{\prod_{u=1}^{r}(a m-u)}
$$


we obtain

$$
\mu_{r}^{\prime}=\sum_{k=0}^{+\infty} \sum_{\ell=0}^{2 k} \sum_{m=1}^{+\infty} \varphi_{k, \ell, m} \int_{0}^{+\infty} x^{r} f_{m}(x ; a) d x=\sum_{k=0}^{+\infty} \sum_{\ell=0}^{2 k} \sum_{m=1}^{+\infty} \varphi_{k, \ell, m} \frac{r !}{\prod_{u=1}^{r}(a m-u)} .
$$

For practical purposes, one can consider a large integer $K$, says $K=50$, and the approximation:

$$
\mu_{r}^{\prime} \approx \sum_{k=0}^{K} \sum_{\ell=0}^{2 k} \sum_{m=1}^{K} \varphi_{k, \ell, m} \frac{r !}{\prod_{u=1}^{r}(a m-u)} ;
$$

therefore, assuming that $a \min (\alpha, 1)>2$, the mean and the variance of $X$ are given by, respectively, $\mu=\mu_{1}^{\prime}$ and $\sigma^{2}=\mu_{2}^{\prime}-\mu^{2}$.

Always under the assumption $a \alpha>r$, the $r$-th central moment of $X$, i.e., $\mu_{r}=E\left[(X-\mu)^{r}\right]$, exists. It is given by

$$
\mu_{r}=\int_{0}^{+\infty}(x-\mu)^{r} f(x ; \alpha, a, b, \lambda) d x .
$$

The standard binomial formula gives

$$
\mu_{r}=\sum_{k=0}^{r}\left(\begin{array}{l}
r \\
k
\end{array}\right)(-1)^{k} \mu^{k} \mu_{r-k}^{\prime}
$$

One can notice that $\sigma^{2}=\mu_{2}$. Additionally, important quantities can be derived, such as the $r$-th cumulant of $X$ defined by

$$
\kappa_{r}=\mu_{r}^{\prime}-\sum_{k=1}^{r-1}\left(\begin{array}{l}
r-1 \\
k-1
\end{array}\right) \kappa_{k} \mu_{r-k}^{\prime}
$$

with $\kappa_{1}=\mu_{1}^{\prime}$, and the Pearson measures of skewness and kurtosis of $X$ defined by $\gamma_{1}=\mu_{3} / \mu_{2}^{3 / 2}$ and $\beta_{2}=\mu_{4} / \mu_{2}^{2}$, respectively.

\subsection{Incomplete Moments}

Let $t \geq 0$ and $r$ be positive integers. Then, the $r$-th incomplete moment of $X$, i.e., $\mu_{r}^{\prime}(t)=$ $E\left(X^{r} 1_{\{X \leq t\}}\right)$ exists and is given by

$$
\mu_{r}^{\prime}(t)=\int_{0}^{t} x^{r} f(x ; \alpha, a, b, \lambda) d x=\sum_{k=0}^{+\infty} \sum_{\ell=0}^{2 k} \sum_{m=1}^{+\infty} \varphi_{k, \ell, m} \int_{0}^{t} x^{r} f_{m}(x ; a) d x .
$$

For given values for $t, \alpha, a, b$ and $\lambda$, a numerical evaluation of the integral term is possible. Incomplete moments appear naturally in several useful quantities. For instance, the mean deviations of $X$ about the mean and the median use $\mu_{1}^{\prime}(t)$; i.e.,

$$
\delta_{\mu}=E(|X-\mu|)=2 \mu F(\mu ; \alpha, a, b, \lambda)-2 \mu_{1}^{\prime}(\mu), \quad \delta_{M}=E(|X-M|)=\mu-2 \mu_{1}^{\prime}(M) .
$$

One can also construct Lorenz and Bonferroni curves, which find applications in economics, insurance and medicine, among others.

\subsection{Weighted Probability Moments}

Let $r$ and $s$ be two positive integers. Then, the $r$, s-th weighted probability moment for $X$, i.e., $\mu_{r, s}^{\prime}=E\left(X^{r} F(X ; \alpha, a, b, \lambda)^{s}\right)$ exists if, and only if, $a \alpha>r$. It is defined by

$$
\mu_{r, s}^{\prime}=\int_{0}^{+\infty} x^{r} f(x ; \alpha, a, b, \lambda) F(x ; \alpha, a, b, \lambda)^{s} d x .
$$


It follows from Proposition 3 applied with $\xi=s$ that

$$
\mu_{r, s}^{\prime}=\sum_{k=0}^{s+1} \sum_{\ell=0}^{+\infty} \sum_{m=0}^{2 \ell} \sum_{q=1}^{+\infty} \phi_{k, \ell, m, q}^{(s)} \int_{0}^{+\infty} x^{r} f_{q}(x ; a) d x=\sum_{k=0}^{s+1} \sum_{\ell=0}^{+\infty} \sum_{m=0}^{2 \ell} \sum_{q=1}^{+\infty} \phi_{k, \ell, m, q}^{(s)} \frac{r !}{\prod_{u=1}^{r}(a q-u)}
$$

The probability weighted moments naturally appear when we deal with some natural statistics, as order statistics. Further details will be presented in Section 5.9.

\subsection{Stress-Strength Reliability Parameter}

Let $X$ and $Y$ be independent random variables having the TIITLIK distribution with the sets of parameters $\left(\alpha_{1}, a_{1}, b_{1}, \lambda_{1}\right)$ and $\left(\alpha_{2}, a_{2}, b_{2}, \lambda_{2}\right)$, respectively. Then, the corresponding stress-strength reliability parameter is defined by $R=P\left(X_{2}<X_{1}\right)$. We should then determine it with several sets of assumptions on the parameter. We begin with a tractable result in that regard.

Proposition 4. Under the above setting, assume that $a_{1}=a_{2}, b_{1}=b_{2}$ and $\lambda_{1}=\lambda_{2}$. Then, we have

$$
R=\frac{\alpha_{2}}{\alpha_{1}+\alpha_{2}}
$$

Proof. By using the definitions of $F\left(x ; \alpha_{2}, a, b, \lambda\right)$ and $f\left(x ; \alpha_{1}, a, b, \lambda\right)$, we get

$$
\begin{aligned}
& R=\int_{0}^{+\infty} F\left(x ; \alpha_{2}, a, b, \lambda\right) f\left(x ; \alpha_{1}, a, b, \lambda\right) d x \\
& =1-\int_{0}^{+\infty}\left\{1-\left[1-(1+x)^{-a}\right]^{2 b}\left\{1+\lambda-\lambda\left[1-(1+x)^{-a}\right]^{b}\right\}^{2}\right\}^{\alpha_{2}} f\left(x ; \alpha_{1}, a, b, \lambda\right) d x \\
& =1-\int_{0}^{+\infty} 2 \alpha_{1} a b(1+x)^{-a-1}\left[1-(1+x)^{-a}\right]^{2 b-1}\left\{1+\lambda-2 \lambda\left[1-(1+x)^{-a}\right]^{b}\right\} \times \\
& \left\{1+\lambda-\lambda\left[1-(1+x)^{-a}\right]^{b}\right\}\left\{1-\left[1-(1+x)^{-a}\right]^{2 b}\left\{1+\lambda-\lambda\left[1-(1+x)^{-a}\right]^{b}\right\}^{2}\right\}^{\alpha_{1}+\alpha_{2}-1} d x \\
& =1-\frac{\alpha_{1}}{\alpha_{1}+\alpha_{2}} \int_{0}^{+\infty} f\left(x ; \alpha_{1}+\alpha_{2}, a, b, \lambda\right) d x=\frac{\alpha_{2}}{\alpha_{1}+\alpha_{2}} .
\end{aligned}
$$

The proof of Proposition 4 is ended.

The result below proposes a more general alternative result.

Proposition 5. Without special assumptions on the parameters, we have

$$
R=1-\sum_{k=0}^{+\infty} \sum_{\ell=0}^{2 k} \sum_{m=0}^{+\infty} \sum_{u=0}^{+\infty} \sum_{v=0}^{2 u} \sum_{w=0}^{+\infty} \varphi_{k, \ell, m}^{(2)} \varphi_{u, v, w}^{(1)} \frac{a_{1} w}{a_{1} w+a_{2} m},
$$

where, for $j \in\{1,2\}, \varphi_{k, \ell, m}^{(j)}=\left(\begin{array}{c}\alpha_{j} \\ k\end{array}\right)\left(\begin{array}{c}2 k \\ \ell\end{array}\right)\left(\begin{array}{c}b_{j}(\ell+2 k) \\ m\end{array}\right)(-1)^{k+\ell+m}\left(1+\lambda_{j}\right)^{2 k-\ell} \lambda_{j}^{\ell}$.

Proof. Owing to Propositions 1 and 2, we have

$$
\begin{aligned}
& R=\int_{0}^{+\infty} F\left(x ; \alpha_{2}, a_{2}, b_{2}, \lambda_{2}\right) f\left(x ; \alpha_{1}, a_{1}, b_{1}, \lambda_{1}\right) d x \\
& =1-\sum_{k=0}^{+\infty} \sum_{\ell=0}^{2 k} \sum_{m=0}^{+\infty} \varphi_{k, \ell, m}^{(2)} \int_{0}^{+\infty} S_{m}\left(x ; a_{2}\right) f\left(x ; \alpha_{1}, a_{1}, b_{1}, \lambda_{1}\right) d x \\
& =1-\sum_{k=0}^{+\infty} \sum_{\ell=0}^{2 k} \sum_{m=0}^{+\infty} \sum_{u=0}^{+\infty} \sum_{v=0}^{2 u} \sum_{w=0}^{+\infty} \varphi_{k, \ell, m}^{(2)} \varphi_{u, v, w}^{(1)} \int_{0}^{+\infty} S_{m}\left(x ; a_{2}\right) f_{w}\left(x ; a_{1}\right) d x
\end{aligned}
$$


Then, by introducing the pdf $f_{*}\left(x ; a_{1} w+a_{2} m, 1\right)$ of the Lomax distribution with parameters $a_{1} w+a_{2} m$ and 1 , we get

$$
\int_{0}^{+\infty} S_{m}\left(x ; a_{2}\right) f_{w}\left(x ; a_{1}\right) d x=\frac{a_{1} w}{a_{1} w+a_{2} m} \int_{0}^{+\infty} f_{*}\left(x ; a_{1} w+a_{2} m, 1\right) d x=\frac{a_{1} w}{a_{1} w+a_{2} m} .
$$

This ends the proof of Proposition 5.

\subsection{Order Statistics}

Since the former study of [24], order statistics found a place as the model of choice for various phenomena dealing with the infima or suprema of random variables. Here, we present some basics on the order statistics in the context of the TIITLIK distribution. Let $X_{1}, \ldots, X_{n}$ be $n$ independent random having the TIITLIK distribution as the common distribution and $X_{i: n}$ be the $i$-th order statistic defined by the $i$-th random variable; i.e., $X_{1: n} \leq X_{2: n} \leq \ldots \leq X_{n: n}$ after rearranging $X_{1}, \ldots, X_{n}$ in an increasing order. Then, the pdf of $X_{i: n}$ is given by

$$
f_{i: n}(x ; \alpha, a, b, \lambda)=\frac{n !}{(i-1) !(n-i) !} \sum_{j=0}^{n-i}\left(\begin{array}{c}
n-i \\
j
\end{array}\right)(-1)^{j} f(x ; \alpha, a, b, \lambda) F(x ; \alpha, a, b, \lambda)^{j+i-1}, \quad x>0 .
$$

Owing to Proposition 3 applied with $\xi=j+i-1$, we obtain the following mixture representation:

$$
f_{i: n}(x ; \alpha, a, b, \lambda)=\sum_{j=0}^{n-i j} \sum_{k=0}^{j+i-1} \sum_{\ell=0}^{+\infty} \sum_{m=0}^{2 \ell} \sum_{q=1}^{+\infty} \rho_{i, j, k, \ell, m, n, q} f_{q}(x ; a),
$$

where $\rho_{i, j, k, \ell, m, n, q}=n ! /[(i-1) !(n-i) !]\left(\begin{array}{c}n-i \\ j\end{array}\right)\left(\begin{array}{c}j+i \\ k\end{array}\right)\left(\begin{array}{c}\alpha k \\ \ell\end{array}\right)\left(\begin{array}{c}2 \ell \\ m\end{array}\right)\left(\begin{array}{c}b(m+2 k) \\ q\end{array}\right)(-1)^{j+k+\ell+m+q+1}(j+i)^{-1}(1+$ $\lambda)^{2 \ell-m} \lambda^{m}$ and $f_{q}(x ; a)$ denotes the pdf of the Lomax distribution with parameters $a q$ and 1.

Let $r$ be a positive integer. Then, the $r$-th moment of $X_{i: n}$ is given by

$$
\mu_{r, i: n}^{\prime}=\sum_{j=0}^{n-i} \sum_{k=0}^{+i-1} \sum_{\ell=0}^{+\infty} \sum_{m=0}^{2 \ell} \sum_{q=1}^{+\infty} \rho_{i, j, k, \ell, m, n, q} \frac{r !}{\prod_{u=1}^{r}(a q-u)} .
$$

Among others, these moments can be used to define the L moments of $X$. Thus, for any $s \in$ $\{1, \ldots, n\}$, the $s$-th $L$ moment of $X$ is given by

$$
\Lambda_{s}=\frac{1}{s} \sum_{d=0}^{s-1}(-1)^{d}\left(\begin{array}{c}
s-1 \\
d
\end{array}\right) \mu_{1, s-d: s}^{\prime} .
$$

We refer the reader to $[25,26]$ for further details on $L$ moments.

\section{Conclusions}

In this paper, we introduced a new, four-parameter lifetime distribution called the type II Topp-Leone (transmuted) inverted Kumaraswamy (TITLIK) distribution. Two applications on practical data sets showed that the TIITLIK distribution provides better fits than several serious competitors, validating its potential in terms of applicability. In order to complete the practical aspect, we provided the main mathematical properties of the new distribution, including asymptotic results, quantile function, Bowley skewness and Moors kurtosis, mixture representations for the probability density and cumulative density functions, ordinary moments, incomplete moments, probability weighted moments, stress-strength reliability and order statistics.

Author Contributions: R.A.Z., F.J., C.C. and M.E. contributed equally to this work. 
Funding: This work was funded by the Deanship of Scientific Research (DSR), King AbdulAziz University, Jeddah, under grant number (DF-284-305-1441).

Acknowledgments: We would like to thank the two reviewers for the valuable and constructive comments. The authors gratefully acknowledge the DSR technical and financial support.

Conflicts of Interest: The authors declare no conflict of interest.

\section{References}

1. Gupta, R.D.; Kundu, D. Exponentiated exponential family: An alternative to Gamma and Weibull distributions. Biom. J. 2001, 43, 117-130. [CrossRef]

2. Eugene, N.; Lee, C.; Famoye, F. Beta-normal distribution and its applications. Commun. Stat.-Theory Methods 2002, 31, 497-512. [CrossRef]

3. Marshall, A.; Olkin, I. A new method for adding a parameter to a family of distributions with applications to the exponential and Weibull families. Biometrika 1997, 84, 641-652. [CrossRef]

4. Zografos, K.; Balakrishnan, N. On families of beta- and generalized gamma-generated distributions and associated inference. Stat. Methodol. 2009, 6, 344-362. [CrossRef]

5. Alizadeh, M.; Emadiz, M.; Doostparast, M.; Cordeiro, G.M.; Ortega, E.M.M.; Pescim, R.R. A new family of distributions: The Kumaraswamy odd log-logistic, properties and applications. Hacet. J. Math. Stat. 2015, 44, 1491-1512. [CrossRef]

6. Cordeiro, G.M.; Alizadeh, M.; Marinho, E.P.R.D. The type I half-logistic family of distributions. J. Stat. Comput. Simul. 2015, 86, 707-728. [CrossRef]

7. Shaw, W.T.; Buckley, I.R. The Alchemy of Probability Distributions: Beyond Gram-Charlier Expansions, and a Skew-kurtotic-normal Distribution from a Rank Transmutation Map. arXiv 2009, arXiv:0901.0434.

8. Alizadeh, M.; Altun, E.; Cordeiro, G.M.; Rasekhi, M. The odd power Cauchy family of distributions: Properties, regression models and applications. J. Stat. Comput. Simul. 2018, 88, 785-805. [CrossRef]

9. Reyad, H.M.; Alizadeh, M.; Jamal, F.; Othman, S.; Hamedani, G.G. The Exponentiated Generalized Topp-Leone-G Family of Distributions: Properties and Applications. Pak. J. Stat. Oper. Res. 2019, 15, 1-24. [CrossRef]

10. Elgarhy, M.; Nasir, M.A.; Jamal, F.; Ozel, G. The type II Topp-Leone generated family of distributions: Properties and applications. J. Stat. Manag. Syst. 2018, 21, 1529-1551. [CrossRef]

11. Jamal, F.; Chesneau, C.; Elgarhy, M. Type II general inverse exponential family of distributions. J. Stat. Manag. Syst. 2019, to appear. [CrossRef]

12. Mohammed, H.F.; Yahia, N. On type II Topp-Leone inverse Rayleigh distribution. Appl. Math. Sci. 2019, 13, 607-615. [CrossRef]

13. Yahia, N.; Mohammed, H.F. The type II Topp-Leone generalized inverse Rayleigh distribution. Int. J. Contemp. Math. Sci. 2019, 14, 113-122. [CrossRef]

14. Abd Al-Fattah, A.M., El-Helbawy, A.A.; Al-Dayian, G.R. Inverted Kumaraswamy distribution: Properties and estimation. Pak. J. Stat. 2017, 33, 37-61.

15. Kumaraswamy, P. A generalized probability density function for double bounded random processes. J. Hydrol. 1980, 46, 79-88. [CrossRef]

16. Iqbal, Z.; Tahir, M.M.; Azeem, S.; Ahmad, M. Generalised inverted Kumaraswamy distribution: Properties and Application. Open J. Stat. 2017, 7, 645-662. [CrossRef]

17. Usman, R.M.; Haq, M.A.U. The Marshall-Olkin extended inverted Kumaraswamy distribution: Theory and applications. J. King Saud Univ.-Sci. 2018. [CrossRef]

18. Reyad, H.; Jamal, F.; Othman, S.; Yahia, N. The Topp-Leone generalized inverted Kumaraswamy distribution: Properties and applications. Asian Res. J. Math. 2019, 13, 1-15. [CrossRef]

19. Ristic, M.; Balakrishnan, N. The gamma-exponentiated exponential distribution. J. Stat. Comput. Simul. 2012, 82, 1191-1206. [CrossRef]

20. Jeong, B.Y.; Seo, Y.A.; Murshed, M.S.; Park, J.S. A three-parameter kappa distribution with hydrologic application: Ageneralized Gumbel distribution. Stoch. Environ. Res. Risk Assess. 2014, 28, 2063-2074. [CrossRef]

21. Aarset, M.V. How to identify bathtub hazard rate. IEEE Trans. Reliab. 1987, 36, 106-108. [CrossRef] 
22. Galton, F. Inquiries into Human Faculty and Its Development; Macmillan and Company, Macmillan: London, UK, 1883.

23. Moors, J.J.A. A quantile alternative for kurtosis. J. R. Stat. Soc. Ser. D (Stat.) 1988, 37, 25-32. [CrossRef]

24. Tippett, L.H.C. On the extreme individuals and the range of samples taken from a normal population. Biometrika 1925, 17, 364-387. [CrossRef]

25. Hosking, J.R.M. L-moments: Analysis and estimation of distributions using linear combinations of order statistics. J. R. Stat. Soc. Ser. B 1990, 52, 105-124. [CrossRef]

26. Hosking, J.R.M. Moments or L moments? An example comparing two measures of distributional shape. Am. Stat. 1992, 46, 186-189. [CrossRef]

(C) 2019 by the authors. Licensee MDPI, Basel, Switzerland. This article is an open access article distributed under the terms and conditions of the Creative Commons Attribution (CC BY) license (http:/ / creativecommons.org/licenses/by/4.0/). 